\title{
STEADY STATE RESPONSE OF A NONLINEAR SYSTEM
}

\section{SUDHANGSHU B. KARMAKAR}

Western Electric Company Whippany, New Jersey 07981 U.S.A.

(Received November 1, 1979)

ABSTRACT. This paper presents a method of the determination of the steady state response for a class of nonlinear systems. The response of a nonlinear system to a given input is first obtained in the form of a series solution in the multidimensional frequency domain. Conditions are then determined for which this series solution will converge. The conversion from multidimensions to a single dimension is then made by the method of association of variables, and thus an equivalent linear model of the nonlinear system is obtained. The steady state response is then found by any technique employed with linear system. KEYWORDS AND PHRASES. Association of variables, Multidimensional frequency domain, Nonlinear transfer function.

1980 MATHEMATICS SUBJECT CLASSIFICATION CODES: $34-00,34 \mathrm{~A} 45$.

1. INTRODUCTION.

This paper considers a nonlinear system described by the equation of the form 


$$
L y+a_{3} y^{3}+a_{5} y^{5}=x(t)
$$

L is a linear operator defined as

$$
L(s)=b_{n} s^{n}+b_{n-1} s^{n-1}+\ldots+b_{0}
$$

$a_{3}, a_{5} ; b_{n}$ are constants. $x(t)$ is the input to the nonlinear system and $y(t)$ is the response to it. Many physical systems encountered in practice can be reiated to above kind of equation. We shall investigate the conditions under which a bounded input to the system will produce a bounded output. This will lead to the stability conditions of the above type of nonlinear system.

\section{ANALYSIS OF A NONLINEAR SYSTEM}

Equation (1.1) can be viewed as follows: Volterra [1] has shown that the response $y(t)$ can be represented as some functional of the input $x(t)$. The following functional expansion was suggested:

$$
y(t)=\int h_{1}\left(t-\tau_{1}\right) x\left(\tau_{1}\right) d \tau_{1}+\iint h_{2}\left(t-\tau_{1}, t-\tau_{2}\right) x\left(\tau_{1}\right) x\left(\tau_{2}\right) d \tau_{1} d \tau_{2}+
$$

We assume a causal system, i.e., $x(t)=0$ for $t<0$. The limits of integrations are $[0, \infty]$. Let

$$
y_{n}(t)=\int_{n-f \circ l d} \ldots \int_{n} h_{n}\left(t-\tau_{1} \ldots t-\tau_{n}\right) x\left(\tau_{1}\right) x\left(\tau_{2}\right) \ldots x\left(\tau_{n}\right) d \tau_{1} d \tau_{2} \ldots d \tau_{n}
$$

Then equation (2.1) can be written as

$$
y(t)=\sum_{n=1}^{\infty} y_{n}(t)
$$

$h_{l}\left(t-\tau_{1}\right)$ is the lst order Volterra Kernel and $h_{n}\left(t-\tau_{1} \ldots t-\tau_{n}\right)$ is the nth order Volterra Kernel. 


\section{DETERMINATION OF THE VOLTERRA KERNELS}

By applying the technique or reversion of algebraic series [2] from (1.1)

we get

$$
y=L^{-1} x-a_{3} L^{-1}\left(L^{-1} x\right)^{3}+L^{-1}\left\{3 a_{3}^{2} L^{-1}\left(L^{-1} x\right)^{5}-a_{5}\left(L^{-1} x\right)^{5}\right\}+
$$

It is clearly understood that $\mathrm{x}$ and $\mathrm{y}$ are both functions of time $t$. Let us define

$$
L^{-1} x \equiv \int h(t-\tau) x(\tau) d \tau
$$

where $h(t)$ is the impulse response of the linear part of the system. Hence from (2.2)

$$
\begin{aligned}
y_{1} & =\int h(t-\tau) x(\tau) d \tau \\
y_{3} & =-a_{3} L^{-1}\left(\int h(t-\tau) x(\tau) d \tau\right)^{3} \\
& =-a_{3} L^{-1} \iiint h\left(t-\tau_{1}\right) h\left(t-\tau_{2}\right) h\left(t-\tau_{3}\right) x\left(\tau_{1}\right) x\left(\tau_{2}\right) x\left(\tau_{3}\right) d \tau_{1} d \tau_{2} d \tau_{3} \\
& =-a_{3} \iiint \int h(t-u) h\left(u-\tau_{1}\right) h\left(u-\tau_{2}\right) h\left(u-\tau_{3}\right) x\left(\tau_{1}\right) x\left(\tau_{2}\right) x\left(\tau_{3}\right) d \tau_{1} d \tau_{2} d \tau_{3} d u
\end{aligned}
$$

Similarly

$$
\begin{array}{r}
y_{5}=3 a_{3}^{2} \int_{7} \ldots \int h(t-v) h(v-u) h\left(u-\tau_{1}\right) \ldots h\left(u-\tau_{5}\right) x\left(\tau_{1}\right) \ldots x\left(\tau_{5}\right) d \tau_{1} \ldots d \tau_{5} d u d v \\
\quad-a_{5} \int_{6} \ldots \int h(t-u) h\left(u-\tau_{1}\right) \ldots h\left(u-\tau_{5}\right) x\left(\tau_{1}\right) \ldots x\left(\tau_{5}\right) d \tau_{1} \ldots d \tau_{5} d u .
\end{array}
$$


Hence by comparing (2.1) and (2.2)

$$
\begin{aligned}
& h_{1}(t-\tau)=h(t-\tau) \\
& h_{2}\left(t-\tau_{1}, t-\tau_{2}\right)=0 \\
& h_{3}=\left(t-\tau_{1}, t-\tau_{2}, t-\tau_{3}\right)=-a_{3} \int h(t-u) h\left(u-\tau_{1}\right) h\left(u-\tau_{2}\right) h\left(u-\tau_{3}\right) d u \\
& h_{4}\left(t-\tau_{1} \ldots t-\tau_{4}\right)=0 \\
& h_{5}\left(t-\tau_{1} \ldots t-\tau_{5}\right)=3 a_{3}^{2} \iint h(t-u) h(v-u) h\left(u-\tau_{1}\right) \ldots h\left(u-\tau_{5}\right) d u d v \\
& \quad-a^{5} \int h(t-u) h\left(u-\tau_{1}\right) \ldots h\left(u-\tau_{5}\right) d u .
\end{aligned}
$$

Higher order kernels can thus be determined by expanding further the equation (3.1).

\section{CONVERGENCY OF THE FUNCTIONAL EXPANSION}

Let

$$
\begin{aligned}
& \max |x(t)| \leq x \\
& 0<t \leq \infty \\
& y_{1} \leq \int|h(t-\tau)||x(\tau) d \tau| \leq \int|h(t)| d t \cdot X=H X
\end{aligned}
$$

where

$$
\begin{aligned}
& \int|h(t)| d t=H<\infty \\
& y_{3} \leq\left|a_{3}\right| \int\left|h\left(t-\tau_{1}\right)\right|\left|x\left(\tau_{1}\right)\right| d \tau_{1} \int\left|h\left(t-\tau_{2}\right)\right|\left|x\left(\tau_{2}\right) d \tau_{2} \int\right| h\left(t-\tau_{3}\right)\left|x\left(\tau_{3}\right)\right| d \tau_{3} \int|h(t-u)| d u .
\end{aligned}
$$

or

$$
y_{3} \leq\left|a_{3}\right|\left(\int|h(t)| d t\right)^{4} x^{3}=\left|a_{3}\right| H^{4} x^{3}
$$


Similarly

$$
y_{5} \leq\left(3 a_{3}^{2} H+\left|a_{5}\right|\right) H^{6} x^{5}
$$

Let

$$
Y=H X+\left|a_{3}\right| H^{4} X^{3}+\left(3 a_{3}^{2} H+\left|a_{5}\right|\right) H^{6} X^{5}
$$

Hence

$$
\left|y_{i}\right|<\left|Y_{i}\right|
$$

Again by applying the technique of reversion of series [ 3 ] it is easy to show that (4.1) is the solution of (4.2)

$$
Y=H X+H\left(\left|a_{3}\right| Y^{3}+\left|a_{5}\right| Y^{5}\right)
$$

and

$$
X=\frac{1}{H} Y-\left|a_{3}\right| Y^{3}-\left|a_{5}\right| Y^{5}
$$

Let $X=X_{1}$ and $Y=Y_{\perp}$ corresponding to the value $\frac{d Y}{d X}=\infty$. Hence if $0<X \leq X_{1}$ then $0<Y \leq Y_{1}$. Therefore in this above region a bounded input will produce a bounded output. $Y_{1}$ can be determined from

$$
5 \mathrm{H}\left|\mathrm{a}_{5}\right| \mathrm{Y}_{1}^{4}+3 \mathrm{H}\left|\mathrm{a}_{3}\right| \mathrm{Y}_{1}^{2}-1=0
$$

corresponding to

$$
\frac{d Y}{d X}=\infty
$$

or

$$
Y_{1}^{2}=\frac{-3 \mathrm{H}\left|\mathrm{a}_{3}\right| \pm \sqrt{9 \mathrm{H}^{2} \mathrm{a}_{3}^{2}+2 \mathrm{OH}\left|\mathrm{a}_{5}\right|}}{1 \mathrm{OH}\left|\mathrm{a}_{5}\right|}
$$


considering only the positive value of $Y_{1}^{2}, Y_{1}$ is found from $Y_{1}=\sqrt{Y_{1}^{2}}$.

From (4.3)

$$
X_{1}=\frac{1}{H} Y_{1}-\left|a_{3}\right| Y_{1}^{3}-\left|a_{5}\right| Y_{1}^{5}
$$

Therefore for $x(t)<x_{1}$, the functional expansion (2.1) will be convergent.

REMARKS:

With the assumed solution of equation (1.1)

$$
\begin{aligned}
y(t) & =\sum_{n=1}^{\infty} \int \ldots \int h_{n}\left(\tau_{1}, \tau_{2} \ldots \tau_{n}\right) x\left(t-\tau_{1}\right) \ldots x\left(t-\tau_{n}\right) d \tau_{1} \ldots d \tau_{n} \\
Y & =\sum_{n=1}^{\infty} c_{n} x^{n}
\end{aligned}
$$

where

$$
C_{n}=\int \ldots \int\left|h\left(\tau_{1}, \tau_{2} \ldots \tau_{n}\right)\right| d \tau_{1} \ldots d \tau_{n}
$$

And for the time invariant system, $C_{n}$ is independent of time [3] which has the radius of convergence $x_{1}$, which in turn implies the convergence of the Volterra series (2.1) for

$$
\begin{gathered}
\max |x(t)|<x_{1} \\
0<t \leq \infty
\end{gathered}
$$

\section{REVIEW OF MULTIDIMENSIONAL TRANSFORM.}

As an analogy to the linear system, the nth order transform of a nonlinear system [4] is defined to be

$$
\left.H_{n}\left(s_{1}, s_{2} \ldots s_{n}\right)=\int \ldots \int h_{n}\left(\tau_{1}, \tau_{2} \ldots \tau_{n}\right) \exp \left[-s_{1} \tau_{1}+s_{2} \tau_{2}+\ldots+s_{n} \tau_{n}\right)\right] d \tau \ldots d \tau_{n}
$$


One method of determining $\mathrm{H}_{n}\left(\mathrm{~s}_{1}, \mathrm{~s}_{2} \ldots \mathrm{s}_{n}\right)$ is the harmonic input method [5]. When the system described by (1.1) is excited with a set of $\mathrm{n}$ unit amplitude exponentials at the noncommensurate frequencies $s_{1}, s_{2} \cdots s_{n}$

$$
x(t)=\exp \left(s_{1} t\right)+\exp \left(s_{2} t\right)+\ldots+\exp \left(s_{n} t\right)
$$

the output will contain exponential components of the form

$$
y(t)=\sum_{n=1}^{\infty} \sum_{M} \frac{{ }_{n}\left(s_{1}, s_{2} \cdots s_{n}\right)}{m_{1} ! m_{2} ! \cdots m_{n} !} \exp \left(m_{1} s_{1}+m_{2} s_{2}+\ldots+m_{n} s_{n}\right)
$$

while $M$ under the summation indicates that for each $n$ the sum is to be taken over all distinct values of $m_{n}$ where $1 \leq m_{1} \leq m_{2} \cdots \leq m_{n}$ such that $m_{1}+m_{2}+\ldots m_{n}=n$.

Thus to determine $\mathrm{H}_{n}\left(s_{1}, s_{2} \ldots s_{n}\right)$ we substitute the values of $x(t)$ and $y(t)$ from (5.2) and (5.3), respectively, in the system equation (1.1) and equate the coefficients of

$$
n ! \exp \left(s_{1}+s_{2}+\ldots+s_{n}\right) t
$$

By substituting the values of $y(t)$ and $x(t)$ from (5.3) and (5.2), respectively, in (1.1) the first three nonzero transfer functions are found to be:

$$
\begin{aligned}
\mathrm{H}_{1}\left(\mathrm{~s}_{1}\right)=\frac{1}{\mathrm{~L}\left(\mathrm{~s}_{1}\right)} & \\
\mathrm{H}_{3}\left(\mathrm{~s}_{1}, \mathrm{~s}_{2}, \mathrm{~s}_{3}\right)= & \frac{-\mathrm{a}_{3}}{\mathrm{~L}\left(\mathrm{~s}_{1}+\mathrm{s}_{2}+\mathrm{s}_{3}\right) L\left(\mathrm{~s}_{1}\right) L\left(\mathrm{~s}_{2}\right) L\left(\mathrm{~s}_{3}\right)} \\
\mathrm{H}_{5}\left(\mathrm{~s}_{1} \ldots \mathrm{s}_{5}\right)= & -\frac{\mathrm{a}_{3}}{\mathrm{~L}\left(\mathrm{~s}_{1}+\mathrm{s}_{2}+\ldots \mathrm{s}_{5}\right) L\left(\mathrm{~s}_{1}+\mathrm{s}_{2}+\mathrm{s}_{3}\right) L\left(\mathrm{~s}_{4}\right) L\left(s_{5}\right)} \\
& +\frac{a_{5}}{L\left(\mathrm{~s}_{1}+\mathrm{s}_{2}+\ldots \mathrm{s}_{5}\right) L\left(\mathrm{~s}_{1}\right) L\left(\mathrm{~s}_{2}\right) \ldots L\left(\mathrm{~s}_{5}\right)}
\end{aligned}
$$


Let $A$ mean associate variables $s_{1}, s_{2} \ldots+s$. Then

$Y(s)=H_{1}(s) X(s)+A\left\{H_{3}\left(s_{1}, s_{2}, s_{3}\right) X\left(s_{1}\right) X\left(s_{2}\right) X\left(s_{3}\right)\right\}+A\left\{H_{5}\left(s_{1} \ldots s_{5}\right) X\left(s_{1}\right) \ldots X\left(s_{5}\right)\right\}+$

Now by applying the Final Value Theorem we get

$$
\lim _{t \rightarrow \infty} y(t)=\lim _{s \rightarrow 0} s Y(s)
$$

Subject to the convergency of (2.1) it is often enough to consider the first two nonzero terms of (5.7) to represent the system in the frequency domain.

\section{EXAMPLE.}

We consider a nonlinear system of the form (1.1) whose linear transfer function is given by

$$
\frac{1}{L(s)}=H(s)=\frac{1}{s^{2}+2 \zeta \omega_{n}^{s}+\omega_{n}^{2}}
$$

Let its impulse response be $h(t)$.

It is easy to see for $\zeta \leq 0, \int|h(t)| d t=H$ is unbounded. Hence the Volterra series expansion (2.1) will be divergent, and the system will not have any bounded steady state response. If $\zeta \geq 1$ it is easy to evaluate $\int|h(t)| d t$ since $s^{2}+2 \zeta \omega_{n}^{2}+\omega_{n}^{2}=0$ will have real roots. If $\mathrm{H}$ is bounded, convergency of the Volterra series can further be investigated. If $0<\zeta<1$ the evaluation of $H$ is a little more involved.

For $0<\zeta<1$

$$
h(t)=\frac{e^{-\zeta \omega_{n} t}}{\omega_{n} \sqrt{1-\zeta^{2}}} \sin \left(\omega_{n} \sqrt{1-\zeta^{2}}\right) t
$$

Let

$$
\alpha=\zeta \omega_{n}, \quad \beta=\omega_{n} \sqrt{1-\zeta^{2}}
$$


hence

$$
h(t)=\frac{e^{-\alpha t}}{\beta} \sin \beta t
$$

We consider the integral

$$
H^{\prime}=\int e^{-\alpha t}|\sin \beta t| d t
$$

Now

$$
\int e^{a x} \sin p x d x=\frac{e^{a x}(a \sin p x-p \cos p x)}{a^{2}+p^{2}}
$$

sin $\beta$ t $<0$ in the intervals $\left\{\frac{\pi}{\beta}, \frac{2 \pi}{\beta}\right\},\left\{\frac{3 \pi}{\beta}, \frac{4 \pi}{\beta}\right\},\left\{\frac{5 \pi}{\beta}, \frac{6 \pi}{\beta}\right\}$, etc. Hence

$$
\begin{aligned}
H^{\prime} & =\frac{\beta}{\alpha^{2}+\beta^{2}}\left[\left\{e^{0}+e^{-m}\right\}+\left\{e^{-m}+e^{-2 m}\right\}+\left\{e^{-2 m}+e^{-3 m}\right\}+\right] \\
& =\frac{\beta}{\alpha^{2}+\beta^{2}}\left[2 e^{0}+2 e^{-m}+2 e^{-2 m}+\ldots-e^{0}\right] ; \quad m=\frac{\alpha \pi}{\beta} \\
H^{\prime} & =\frac{\beta}{\alpha^{2}+\beta^{2}}\left[\frac{2}{1-e^{-n}}-1\right]=\frac{\beta}{\alpha^{2}+\beta^{2}} \cdot \frac{1+e^{-m}}{1-e^{-m}}
\end{aligned}
$$

Now $-\operatorname{coth}(-u)=\operatorname{coth} u=\frac{1+e^{-2 u}}{1-e^{-2 u}}$, hence

$$
H^{\prime}=\frac{\beta}{\alpha^{2}+\beta^{2}} \operatorname{coth}\left(\frac{m}{2}\right)
$$

Therefore,

$$
H=\frac{1}{\omega_{n}^{2}} \operatorname{coth}\left(\frac{\pi \zeta}{2 \sqrt{1-\zeta^{2}}}\right)
$$


As a numerical example we consider the following system:

$$
\ddot{y}+\dot{y}+3 y+\tanh y=A \cos \omega t
$$

By expanding tanh $\mathrm{y}$ and by retaining the first three terms we get

$$
\ddot{y}+\dot{y}+4 y-\frac{1}{3} y^{3}+\frac{2}{15} y^{5}=A \cos \omega t
$$

The above approximation is quite valid if $y<1$. Here

$$
\zeta=\frac{1}{4}, \quad \omega_{n}=2
$$

Hence

$$
\begin{aligned}
H & =\frac{1}{4} \operatorname{coth}\left(\frac{\pi \times 0.25}{2 \times 0.96825}\right)=0.64984 \\
Y_{1}^{2} & =\frac{-3 \times 0.64984 \times \frac{1}{3} \pm \sqrt{9 \times(0.64984)^{2} \cdot \frac{1}{9}+20 \times 0.6498 \times \frac{2}{15}}}{10 \times 0.64984} \\
& =+0.94432 \text { hence } Y_{1}=0.9717658 \\
X_{1} & =\frac{0.9717658}{0.64984}-\frac{1}{3}(0.9717658)^{3}-\frac{2}{15}(0.9717658)^{5} \\
& =1.49539-0.305888-0.1155438=1.07395
\end{aligned}
$$

Hence the system will have a steady response for $|A \cos \omega t|<1.07395$. Response of the linear part $=\lim _{s \rightarrow 0} \frac{1}{s^{2}+s+4} \cdot \frac{A^{2}}{s^{2}+w^{2}}=0$. It is not necessary to determine the response of the nonlinear parts because being convergent, the other terms of the Volterra expansion can not exceed in absolute value of the linear term. Hence the system will be stable for $|x(t)|<|1.07395 \cos \omega t|$. If $x(t)=A$, then for $A<1.07309$, the approximate value of the steady state response is given by 


$$
\begin{aligned}
& \lim _{t \rightarrow \infty} y(t)=\lim _{t \rightarrow \infty} y_{1}(t)+\lim _{t \rightarrow \infty} y_{3}(t) \\
& \lim _{t \rightarrow \infty} y_{1}(t)=\lim _{s_{1} \rightarrow 0} \frac{s_{1}}{s_{1}^{2}+2 \zeta \omega_{n} s_{1}+\omega_{n}^{2}} \cdot \frac{A}{s_{1}}=\frac{A}{\omega_{n}^{2}}
\end{aligned}
$$

In order to determine $\lim _{t \rightarrow \infty} y_{3}(t)$ we first obtain $Y_{3}\left(s_{1}, s_{2}, s_{3}\right)$

$$
Y_{3}\left(s_{1}, s_{2}, s_{3}\right)=H_{3}\left(s_{1}, s_{2}, s_{3}\right) X\left(s_{1}\right) X\left(s_{2}\right) X\left(s_{3}\right)
$$

From (5.5)

$$
Y_{3}\left(s_{1}, s_{2}, s_{3}\right)=-\frac{a_{3}}{L\left(s_{1}+s_{2}+s_{3}\right) L\left(s_{1}\right) L\left(s_{2}\right) L\left(s_{3}\right)} \frac{A^{3}}{s_{1} s_{2} s_{3}}
$$

Where

$$
L(s)=s^{2}+2 \zeta \omega_{n} s+\omega_{n}^{2}
$$

Now by the technique of association of variables $[7,8,9] \mathrm{Y}_{3}\left(\mathrm{~s}_{1}, \mathrm{~s}_{2}, \mathrm{~s}_{3}\right)$ is translated in one frequency domain, thus we obtain

$$
Y_{3}(s)=-\frac{A^{3} a_{3}}{\left(s^{2}+2 \zeta \omega_{n} s+\omega_{n}^{2}\right)}\left[\frac{1}{\omega_{n}^{4} s\left(s^{2}+2 \zeta \omega_{n}^{2}+\omega_{n}^{2}\right)}+\frac{1}{A_{1}}+\ldots \frac{1}{A_{8}}\right]
$$

$\frac{1}{A_{1}}, \frac{2}{A_{2}} \ldots \frac{1}{A_{8}}$ are functions of $\left(s, \zeta, \omega_{n}\right)$ but do not contain any factor of the form $\frac{1}{\mathrm{~s}}$. Hence

$$
\begin{aligned}
\lim _{t \rightarrow \infty} y_{3}(t)= & -\lim _{s \rightarrow 0} \frac{s A^{3} a_{3}}{\left(s^{2}+2 \zeta \omega_{n}^{2}+\omega_{n}^{2}\right)} \cdot \frac{1}{\omega_{n}^{4} s\left(s^{2}+2 \zeta \omega_{n} s+\omega_{n}^{2}\right)} \\
& +\lim _{s \rightarrow 0} s\left(\frac{1}{A_{1}}+\ldots \frac{1}{A_{8}}\right)
\end{aligned}
$$




$$
\lim _{t \rightarrow \infty} y_{3}(t)=-\frac{a_{3} A^{3}}{\omega_{n}^{8}}+0
$$

The steady state response of the system is approximately given by

$$
y_{s s}=\frac{A}{\omega_{n}^{2}}\left(1-\frac{A a_{3}}{\omega_{n}^{6}}\right)
$$

For

$$
\begin{aligned}
& a_{3}=-\frac{1}{3}, \quad \omega_{n}^{2}=4, \quad A=1 \\
& y_{S S}=\frac{1}{4}\left(1+\frac{1}{3.64}\right)=\frac{1}{4}+\frac{1}{768}
\end{aligned}
$$

If $x(t)=\delta(t)$. Again by associating the variables [8] we get

$$
y_{s s}=\lim _{s \rightarrow 0} \frac{s}{s^{2}+2 \zeta \omega_{n} s+\omega_{n}^{2}}+\lim _{s \rightarrow 0}\left(\frac{1}{B_{1}}+\frac{1}{B_{2}}+\frac{1}{B_{3}}\right)=0
$$

Since $\frac{1}{B_{1}}, \frac{1}{B_{2}}, \frac{1}{B_{3}}$ do not contain any factor of the form $\frac{1}{s}$.

ACKNOWLEDGMENT: The author gratefully acknowledges the assistance of fered to him by Prof. J. F. Barrett, Department of Mathematics, Eindhoven University of Technology, The Netherlands.

\section{REFERENCES}

1. Volterra, V., Theory of Functionals and of Integral and Integro Differential Equations, Dover Publications, New York, 1959.

2. Hodgman, C. D., Standard Mathematical Tables, Chemical Rubber Publishing Company, Cleveland, Ohio, 1959.

3. Barrett, J. F., The series-reversion method for solving forced nonlinear differential equations, Math. Balkanica, Vol. 4.9, 1974, pp. 43-60. 
4. Barrett, J. F., The Use of Functionals in the Analysis of Nonlinear Physical Systems, J. Electronics and Control 15, 567-615, 1963.

5. Kuo, Y. L., Frequency-Domain Analysis of Weakly Nonlinear Networks, Circuits and Systems, (IEEE) Vol. 11, No. 4, 2-8, August 1977.

6. Bedrosian, E., Rice, S. O., Output Properties of Volterra Systems (Nonlinear Systems with Memory) Driven by Harmonic and Gaussian Inputs, Proc. IEEE 59 1688-1707, December 1971.

7. Dorf, R. C., Modern Control Systems, Addison-Wesley Publishing Company, Reading, Massachusetts, 1967.

8. George, D. A., Continuous Nonlinear Systems MIT Research Laboratory of Electronics, Technical Report 355, July 24, 1959.

9. Lubbock, J. K., Bansal, V. S., Multidimensional Laplace transforms for solution of nonlinear equation, Proc. IEEE, Vol. 116 (12) 2075-2082, December 1969.

10. Koh, E. L., Association of variables in n-dimensional Laplace transform, Int. J. Systems Sci., Vol. 6 (2). 


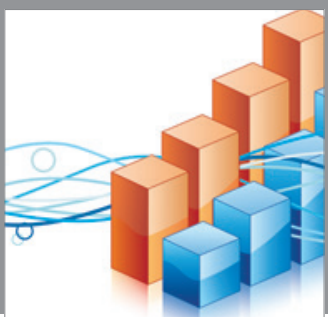

Advances in

Operations Research

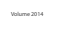

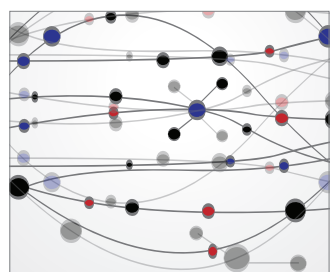

\section{The Scientific} World Journal
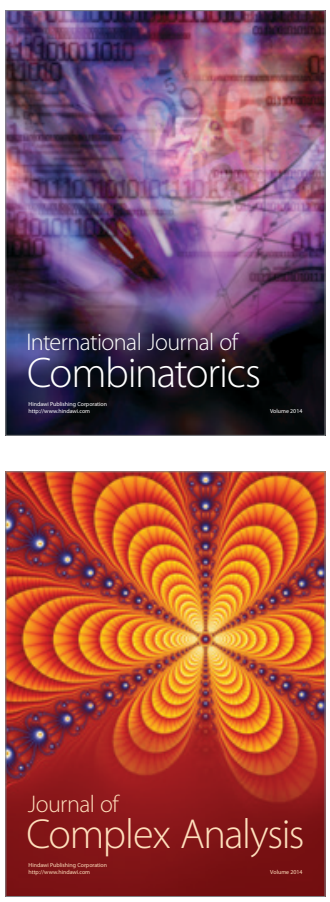

International Journal of

Mathematics and

Mathematical

Sciences
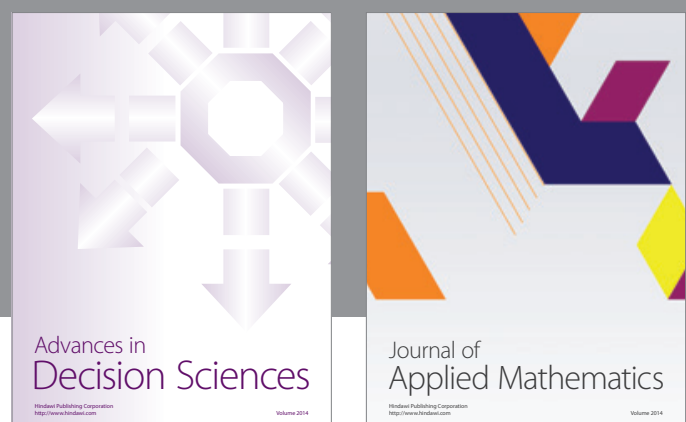

Journal of

Applied Mathematics
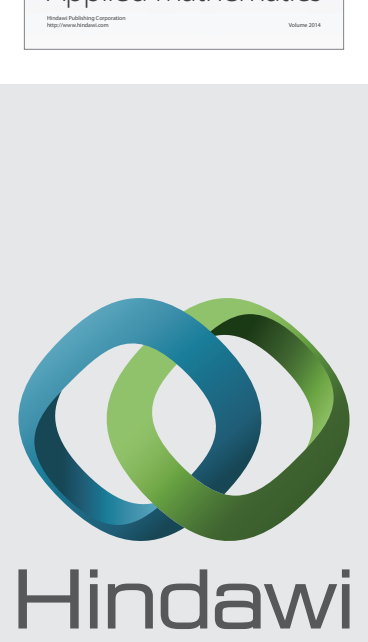

Submit your manuscripts at http://www.hindawi.com
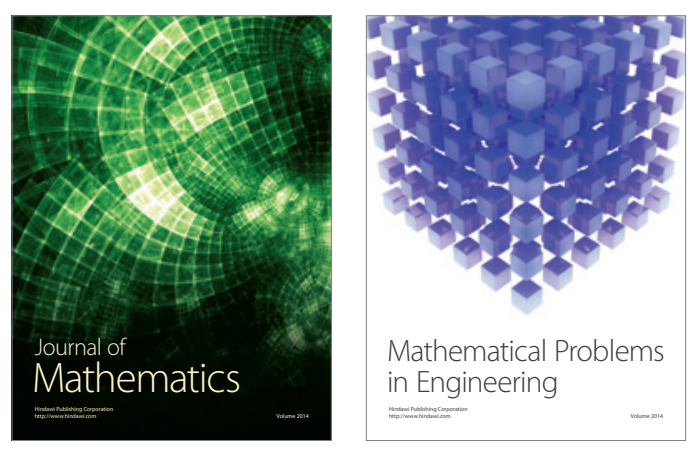

Mathematical Problems in Engineering
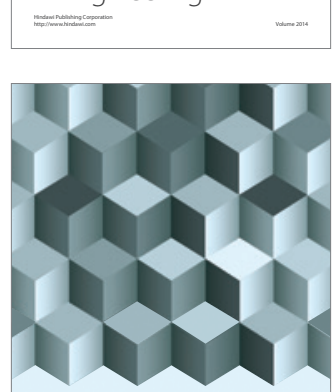

Journal of

Function Spaces
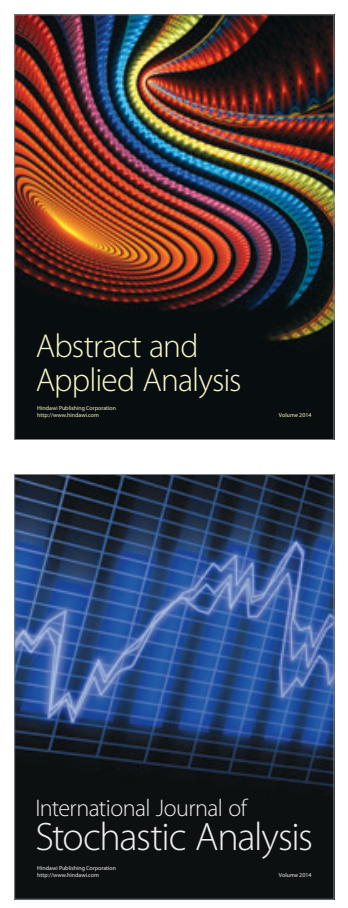

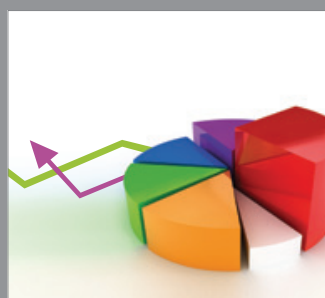

ournal of

Probability and Statistics

Promensencen
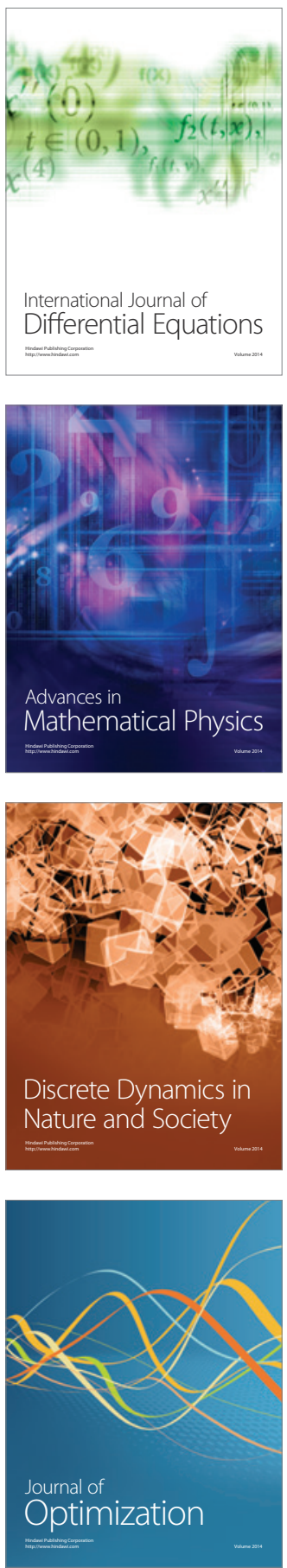